\title{
FIRST WORKSHOP ON MULTIMODAL E-COACHES
}

PREPRINT

Leonardo Angelini, Mira El Kamali, Elena Mugellini, Omar Abou Khaled

HumanTech Institute, University of Applied Sciences Western Switzerland HES-SO, Fribourg, Switzerland

\{leonardo.angelini, mira.elkamali, elena.mugellini, omar.aboukhaled\}@hes-so.ch

Yordan Dimitrov, Vera Veleva, Zlatka Gospodinova, Nadejda Miteva, Richard Wheeler Balkan Institute for Labour and Social Policy, Sofia, Bulgaria

jordandim@bilsp.org, vera.veleva@bilsp-pc.org, zlatka@bilsp.org

n.miteva@bilsp-pc.org, rwprivate@gmail.com

Zoraida Callejas, David Griol, Kawtar Benghazi, Manuel Noguera

Dept. Software Engineering, University of Granada, Granada, Spain

\{zoraida, dgriol, benghazi, mnoguera\}@ugr.es

Panagiotis Bamidis, Evdokimos Konstantinidis, Despoina Petsani University of Thessaloniki, Thessaloniki, Greece

bamidis@med.auth.gr, evdokimosk@gmail.com, despoinapets@gmail.com

Andoni Beristain Iraola

Vicomtech Foundation, Basque Research and Technology Alliance Donostia-San Sebastián, Spain aberistain@vicomtech.org

Dimitrios I. Fotiadis

Unit of Medical Technology and Intelligent Information Systems, Department of Materials Science and Engineering, University of Ioannina, Greece

fotiadis@uoi.gr

\section{Gérard Chollet}

Intelligent Voice, London, UK gerard.chollet@intelligentvoice.com

María Inés Torres

Dept. of Electrical and Electronics, University of the Basque Country, Leioa, Spain manes.torres@ehu.eus

\section{Anna Esposito}

University of Campania Luigi Vanvitelli, Caserta, Italy iiass.annaesp@tin.it

\section{Hannes Schlieter}

Dresden University of Technology, Dresden, Germany hannes.schlieter@tu-dresden.de 
This is a pre-print version of the paper:

Leonardo Angelini, Mira El Kamali, Elena Mugellini, Omar Abou Khaled, Yordan Dimitrov, Vera Veleva, Zlatka Gospodinova, Nadejda Miteva, Richar Wheeler, Zoraida Callejas, David Griol, Kawtar Benghazi, Manuel Noguera, Panagiotis Bamidis, Evdokimos Konstantinidis, Despoina Petsani, Andoni Beristain Iraola, Dimitrios I. Fotiadis, Gérard Chollet, Inés Torres, Anna Esposito, and Hannes Schlieter. 2020. First Workshop on Multimodal e-Coaches. In Proceedings of the 2020 International Conference on Multimodal Interaction (ICMI'20). Association for Computing Machinery, New York, NY, USA, 890-892. https://doi.org/10.1145/3382507.3420056

This preprint follows ACM policy: "Authors who publish with ACM have the freedom to post peer-reviewed pre-print versions of their papers to personal websites and institutional repositories. They can add a single-click link to their final published papers, and re-use any portion of their published work with the inclusion of a citation and DOI link. Authors can also post on any repository legally mandated by the agency funding the research on which the work is based, and on any non-commercial repository or aggregation that does not duplicate ACM tables of contents/substantially duplicate an ACM-copyrighted volume or issue" (https://authors.acm.org/author-resources/author-rights.) 


\begin{abstract}
e-Coaches are promising intelligent systems that aims at supporting human everyday life, dispatching advice through different interfaces, such as apps, conversational interfaces and augmented reality interfaces. This workshop aims at exploring how e-coaches might benefit from spatially and timemultiplexed interfaces and from different communication modalities (e.g., text, visual, audio, etc.) according to the context of the interaction.
\end{abstract}

Keywords Mental health $\cdot$ e-mental health $\cdot$ engagement $\cdot$ adherence $\cdot$ attrition $\cdot$ e-health $\cdot$ m-health $\cdot$ digital health intervention $\cdot$ e-coaches

\title{
1 Workshop Motivation
}

Virtual coaches, also known as e-coaches, are interactive systems able to observe, monitor, listen to and proactively give advice to the user [1], for different purposes, generally linked to the user well-being. Providing personalized coaching in different well-being domains to a large number of people would be particularly challenging, since multi-domains human experts might be difficult to find and the cost of a large-scale intervention might be not sustainable for most countries. E-coaches, instead, are able to monitor and dispatch personalized advice in a convincing manner, taking advantage of devices that the users might already have at their disposal. Indeed, the diffusion and democratization of the Internet of Things (IoT) enables new interaction scenarios for the e-coaches, who can monitor user's daily life and provide advice through multiple interfaces. At the same time, e-Coaches will be able to replace or complement human coaches only if they can show very advanced behaviours: Kamphorst [2] highlighted that e-coaches should have a social ability, i.e., being able to converse with and learn about the user, should be credible and support behaviour change, proactively giving suggestions to the user in a context-aware manner, supporting the user's self-reflection and his/her planning ability.

The European Commission has recently funded 8 European projects under the call SC1-PM15-2017. These projects aim at designing and developing innovative virtual coaches able to support and accompany older adults in their daily life. Each project takes advantage of different interfaces for monitoring user well-being and for interacting with the user. For example, NESTORE [3, 4] uses smart sensors (bracelet, scale, sleep monitor, beacons) to monitor the user well-being and a conversational agent (both in form of chatbot and tangible vocal assistant), apps (including a serious game and a web social platform) to coach the user. SAAM system spans from unobtrusive data collection through data processing and situation assessment of the social behaviour to selection and rendering the most appropriate coaching actions to the elderly person including through members of their social circles. CAPTAIN [5] uses unobtrusive monitoring (3D depth sensors) to detect user's movement inside the house and perform gait analysis, micro-projections whenever and wherever needed to deliver information, tangible environment and conversational modalities for receiving user's input. HOLOBALANCE is addressed to citizens with balance disorders and its aim is to achieve short and long-term behavioural change by means of increased adherence to balance rehabilitation regimes and adoption of a healthier lifestyle, respectively. This system has developed and is evaluating a radically new virtual coach with motivational elements, consisting of a) a hologram based surrogate balance physiotherapist interacting with the user based on the evaluation of performance with sensors (IMUs, insoles, depth camera), b) augmented reality cognitive games and exergames, c) a tool for auditory training as well as d) a physical activity planning app. vCare uses a Virtual Coach as a primary mode of communication and motivation for patients continuing their rehabilitation care after an acute incident at home. The coach is fed by a multitude of sensors, machine learning elements, coaching services, serious games and personsalised clinical pathways in order to optimally support the patient's recovery. MENHIR, funded under the call H2020-MSCA-RISE-2018, explores the possibilities of conversational technologies to understand, promote and protect mental health and assist people with anxiety and mild depression manage their conditions. MENHIR uses cross-modal analysis of the user interactions (paralinguistic and dialogue features) in order to recognize the users' emotional state and anxiety levels and generate adequate dialogue management strategies.

The conjunction of e-Coaches and the IoT enables new interaction perspectives for the users. Indeed, coaching users across a plethora of interfaces and interaction modalities open new interaction scenarios and new challenges for the HCI community. Typical questions that arise are the following. How to maintain the e-coach identity and coherence across different interfaces? How to sustain trust and empathy across different interfaces? How to intelligently dispatch advice across interfaces that are distributed in spaces? Which modalities should be used in the different life settings? Can multimodal interaction increase the accessibility of the e-coach? How to make e-Coaches as unobtrusive as possible? How to maintain adherence to e-coaching programmes? The first workshop on multimodal e-Coaches aims at being a venue for discussing these questions and other $\mathrm{HCI}$ challenges that arise with e-Coaches distributed across multiple interfaces and devices. 


\section{Workshop Format}

The workshop aims at being an open venue for researchers and practitioners interested in discussing the challenges associated to the interaction with e-Coaches across multiple modalities and/or devices. We wish to take a snapshot of the current state of the art on multimodal interaction with e-Coaches and to discuss future pathways of research in this domain.

To this purpose, in the morning workshop participants will have the opportunity to present their research and to discuss it with the other participants. Starting from the topics discussed in the morning, the afternoon will be devoted to hands-on and brainstorming activities for the design of multimodal interfaces for distributed e-Coaches. The results of these activities will be discussed to possible draw guidelines for the designs of multimodal e-coaches.

More information about the workshop program and the accepted papers can be found on the workshop website: https://multimodal-ecoches.nestore-coach.eu/

\section{Expected Outcomes and Impact}

The workshop aims to establish itself as the premiere shared forum for multimodal e-Coaches. We aim at involving researchers and practitioners, particularly those who are seeking new insights for understanding how e-Coaches will evolve in the future. We want to create a multidisciplinary space where researchers can discuss how these technologies should evolve to be integrated with existing practices, individual needs and levels of technical expertise, effectively supporting a variety of people's tasks and goals in their everyday lives. In particular, the workshop aims at discussing multimodal e-Coaches that can be embedded in the daily routines of the users through wearable interfaces, embodied representations of the e-Coach (e.g., smart companions), conversational agents and distributed augmented reality interfaces. The long-term objective of the workshop is to build a community interested in sharing ideas on multimodal e-coaches. This includes researchers with different backgrounds, from engineers and computer scientists, to designers, social scientists, ethnographers and psychologists.

We expect to gather at the workshop 20-30 researchers and practitioners interested in multimodal interaction with e-Coaches and to receive 8-10 position papers/early stage results to be presented at the workshop during the morning sessions. The organizers of the workshop will all also present the results of the EU projects NESTORE, SAAM, CAPTAIN, HOLOBALANCE, EMPATHIC, MENHIR, vCare. At the end of the morning we will be able to draw a picture of the current solutions for multimodal interaction with e-coaches. The afternoon discussion and activities will be devoted to solve the open challenges in this domain. At the end of the workshop, we expect to derive a set of guidelines for the design of multimodal interaction with e-coaches that can be shared as a white paper or a further publication with the HCI community. The workshop will also allow to gather and federate a community of researchers and practitioners interested in multimodal e-coaches, hopefully interested in organizing other events related to this topic and in collaborating in further research projects.

\section{Workshop Topics}

The workshop aims at gathering contributions for the design of e-Coaches that are embedded in the user's daily life through multimodal interfaces. This is a non-exhaustive list of topics for the workshop position papers:

- Spatially distributed interfaces for e-Coaches;

- Augmented reality and voice interfaces for e-Coaches;

- Text and voice conversational agents for coaching;

- Embodied and empathic virtual coaches; 
- Talking companions;

- Data collection and presentation from e-coaches;

- Intelligent context-aware fission of coaching advices;

- Multimodal fusion of inputs for e-Coach monitoring and interaction;

- User modelling for e-coaching;

- Trust and user rapport;

- Particular applications of e-coaches: healthy living, well-being, etc.;

- Context-aware conversations and interactions with e-coaches;

- Long-term usage of multimodal e-Coaches;

- Design methods for multimodal e-Coaches;

- Unobtrusive ambient sensing feeding e-Coaches with data.

\section{Acknowledgements}

The NESTORE, SAAM, CAPTAIN, HOLOBALANCE, EMPATHIC, MENHIR, vCare projects are supported by the European Commission under the Horizon 2020 programmes SC1-PM-15-2017 H2020-MSCA-RISE-2018, and H2020-1.3.3, respectively through the project grants N.769643, 769661, 769830, 769574, 769872, 823907, 769807. The authors want to thank their respective Consortia. The opinions expressed in this paper are those of the authors and are not necessarily those of the project partners or the European Commission.

\section{References}

[1] Oresti Banos and Christopher Nugent. E-coaching for health. Computer, 51(3):12-15, 2018.

[2] Bart A Kamphorst. E-coaching systems. Personal and Ubiquitous Computing, 21(4):625-632, 2017.

[3] Leonardo Angelini, Elena Mugellini, Omar Abou Khaled, Christina Röcke, Sabrina Guye, Simone Porcelli, Alfonso Mastropietro, Giovanna Rizzo, Noemi Boqué, Josep M del Bas, et al. The nestore e-coach: accompanying older adults through a personalized pathway to wellbeing. In Proceedings of the 12th ACM International Conference on PErvasive Technologies Related to Assistive Environments, pages 620-628, 2019.

[4] Mira El Kamali, Leonardo Angelini, Maurizio Caon, Giuseppe Andreoni, Omar Abou Khaled, and Elena Mugellini. Towards the nestore e-coach: a tangible and embodied conversational agent for older adults. In Proceedings of the 2018 ACM International Joint Conference and 2018 International Symposium on Pervasive and Ubiquitous Computing and Wearable Computers, pages 1656-1663, 2018.

[5] Evdokimos I Konstantinidis, Despoina Petsani, Giuseppe Conti, Antonis Billis, Valentina Conotter, Guillaume Chican, Tim Llewellynn Lorenzo, Alejandro Rivero Rodríguez, Santiago Hors-Fraile, Andoni Beristain, et al. A new approach for ageing at home: The captain system. In MedInfo, pages 1704-1705, 2019. 